\title{
Perceptions of genetic variant reclassification in patients with inherited cardiac disease
}

\author{
Eugene K. Wong ${ }^{1} \cdot$ Kirsten Bartels $^{2}$ - Julie Hathaway ${ }^{2}$ Charlotte Burns $s^{3,4,5} \cdot$ Laura Yeates $^{3,4,5}$. \\ Christopher Semsarian ${ }^{3,4,5} \cdot$ Andrew D. Krahn ${ }^{2} \cdot$ Alice Virani ${ }^{1} \cdot$ Jodie Ingles $\mathbb{D}^{3,4,5}$
}

Received: 10 November 2018 / Revised: 16 January 2019 / Accepted: 1 March 2019 / Published online: 21 March 2019

(c) European Society of Human Genetics 2019

\begin{abstract}
Interpretation of sequence variants is an ongoing challenge and new approaches aim to increase stringency. The reclassification of variants has the potential to alter medical management and elicit psychosocial consequences for patients. The perspective of patients with an inherited cardiac disease and a clinically significant variant reclassification was explored through semi-structured phone interviews. Participants were recruited from two specialized multidisciplinary centers in Canada and Australia. Qualitative analysis was performed through a thematic analysis approach. Fifteen participants were interviewed, including $9(60 \%)$ with an inherited cardiomyopathy and $6(40 \%)$ with an inherited arrhythmia syndrome. Six $(40 \%)$ patients had a classification upgrade, while $9(60 \%)$ had a downgrade. Four major themes emerged: (1) reactions towards the reclassified variant; (2) impact on decision-making; (3) perception of the reclassification process; and (4) improvement of the reclassification process. Many patients adjusted to the reclassification, however some misunderstood the implications, impacting their responses and decision-making. In conclusion, careful discussion with patients about uncertainty and the potential for reclassification are crucial to ensure a deeper understanding of the outcome of genetic testing and impact on families.
\end{abstract}

\section{Introduction}

Inherited cardiac diseases include both cardiomyopathies and arrhythmia syndromes. They are genetically heterogeneous in terms of the alleles and loci that can cause diseases, with numerous genes implicated [1]. With the increase in the number of genes now routinely included on

These authors contributed equally: Alice Virani, Jodie Ingles

Jodie Ingles

j.ingles@centenary.org.au

1 Department of Medical Genetics, University of British Columbia, Vancouver, BC, Canada

2 BC Inherited Arrhythmia Program, Vancouver, BC, Canada

3 Agnes Ginges Centre for Molecular Cardiology at Centenary Institute, The University of Sydney, Sydney, NSW, Australia

4 Sydney Medical School, Faculty of Medicine and Health, The University of Sydney, Sydney, NSW, Australia

5 Department of Cardiology, Royal Prince Alfred Hospital, Sydney, NSW, Australia cardiac gene panels, coupled with the decrease in sequencing cost, access to and uptake of genetic testing are greater than ever. While the expansion in the number of genes being tested in a panel may increase the diagnostic yield in certain cardiac diseases, it also increases the detection of variants of uncertain significance (VUS) [2-4]. Genetic results are probabilistic in nature, where evidence for or against disease causation is weighted to make a classification [4]. Uncertainty is inherent to the process, and as the scientific and medical communities continually refine methods for interpreting genomic variation, review and reclassification of variants are necessary.

The American College of Medical Genetics and Genomics and Association of Medical Pathologists (ACMG/ AMP) released revised criteria for interpreting sequence variants, which have helped in standardizing the approach to classification [5]. Most significantly, the release of population data via the Exome Aggregation Consortium (ExAC) [6] and Genome Aggregation Database (gnomAD) has spurred the reclassification of numerous variants by showing that collectively, rare variation occurs frequently in individuals. Conversely, these databases have also shown that many variants are too common to be truly disease- 
causing, which has been a major contributor to variant reclassification. In the setting of inherited cardiac diseases, $36 \%$ of variants were reclassified from their clinical reports when the ACMG/AMP guidelines were later applied [7]. Another study showed $10 \%$ of hypertrophic cardiomyopathy $(\mathrm{HCM})$ variants were reclassified when applying more rigorous pathogenicity criteria [8]. Variant reclassification can in some cases pose a clinical dilemma, as medical therapy and surveillance may have been already initiated, though for the large part medical management decisions are based on the presence of a clinical phenotype.

Importantly, the impact extends beyond the proband to the at-risk relatives. Clinical screening advice is contingent on the initial interpretation of the variant, and there is potential for inappropriate discharge from follow up, or unnecessary screening and worry for those relatives who undergo cascade genetic testing of an incorrectly classified variant. Reclassification may also have an important impact on reproductive choices, some of which may be irreversible. In the cancer setting, some patients continued to pursue major medical therapies and interventions, such as a riskreducing surgery, based on an initial VUS result, which was later reclassified to a benign variant [9]. Uncertainty can be challenging to convey and can lead to misunderstanding [10], as well as eliciting both positive and negative affective responses, depending on patients interpretation of the result $[11,12]$. A paucity of literature exists on patients' perspectives of receiving a variant reclassification. As such, we sought to explore the impact of receiving a variant reclassification in the setting of inherited cardiac diseases.

\section{Materials and methods}

\section{Study sample and recruitment}

Participants were recruited at two international sites: the $\mathrm{BC}$ Inherited Arrhythmia Program and the HCM Clinic (St. Paul's Hospital, Vancouver, Canada) and the HCM and Genetic Heart Diseases Clinic (Royal Prince Alfred Hospital, Sydney, Australia). Both sites are specialized multidisciplinary clinics, where patients are seen and followed long-term by cardiologists and genetic counselors with disease-specific expertise.

Eligibility included probands that (1) underwent genetic testing for their suspected inherited arrhythmia or cardiomyopathy and had a genetic variant returned, and (2) received a clinically meaningful upgrade or downgrade of their variant, such as likely pathogenic (LP)/pathogenic (P) to variant of uncertain significance (VUS)/likely benign (LB) or vice versa, and (3) sufficient English speaking skills as self-reported by the participant. Consistent with the $\mathrm{ACMG} / \mathrm{AMP}$ revised criteria, we refer $\mathrm{P}$ and LP variants as those that have a high likelihood of affecting gene function and thus causing disease. A LB variant is defined as a variant that is unlikely to affect gene function and cause disease. We define a clinically meaningful reclassification as one that would have implications on surveillance and medical therapy recommendations for the proband and atrisk relatives. As per clinical practice in each clinic setting, probands were notified of their result reclassification either during their routine follow-up by the genetic counselor and cardiologist, or were contacted by phone by the genetic counselor. Following disclosure of the reclassification, an amended results letter and the revised genetic report were sent to the patient.

Participants were invited through a purposive sampling strategy by the clinical genetic counselors, by identifying those that met the above eligibility criterion. A retrospective chart review of patients that have been seen at each site was also conducted to capture additional participants. At the Australian site, participants were recruited by phone. At the Canadian site, participants were approached by email or letter mail, followed by a reminder phone call.

\section{Study design}

We conducted semi-structured interviews in order to capture the different experiences of receiving a variant reclassification. The interview guide was designed from a literature review and from discussion amongst the research team. It comprised of questions that addressed patients' understanding of their genetic results, the psychosocial impact of the reclassification process and result, and improvement of the patient experience towards receiving a reclassification. Phone recorded interviews ranging from 20 to $60 \mathrm{~min}$ were conducted by the primary author, who had no prior relationship with any research participants. Demographic information was obtained prior to beginning the recorded interview. Interviews continued until thematic saturation was obtained.

\section{Data analysis}

De-identified interviews were transcribed verbatim by the primary author and study volunteers. Thematic analysis was used to analyze the transcripts [13]. The primary author was responsible for coding all transcripts. A code list was generated by the primary author through coding an initial subset of transcripts, and was reviewed with the research team to ensure agreement with the codes. The list was revised through an iterative process as additional interview transcripts were analyzed. In order to address possible cultural influences on themes, which may arise from recruitment at two different countries, all team members took part in coding two transcripts from each country. To 
ensure inter-coder consistency and to validate the master code list, four transcripts were reviewed and coded by the whole team. Any coding discrepancies were discussed until a consensus was reached for the final code. The master code list was then used to derive overarching themes.

\section{Results}

There were 21 individuals invited to participate in the study. Five individuals did not respond to the invitation, and one declined to participate. A total of 15 participants (eight Australian and seven Canadian) completed the interview, resulting in a total response rate of $71.4 \%$ (15/21) (Table 1). Nine $(60 \%)$ were diagnosed with an inherited cardiomyopathy, and six (40\%) with an inherited arrhythmia syndrome. Six $(40 \%)$ patients had a classification upgrade, while nine $(60 \%)$ had a downgrade. Participants were primarily of Caucasian ethnicity (93.3\%), females (73.3\%), more than 40 years of age (60.1\%) and had a family history of the cardiac condition or sudden cardiac death $(60 \%)$. The mean time from when an initial result was reclassified was $28.5 \pm 23.5$ months. The mean time of the interview after a reclassification was $19.7 \pm 12.3$ months.

\section{Participant recall of the reclassified variant}

In general, participants were unable to recall the technical terminology for their reclassified result. However, their understanding of their reclassification was demonstrated through explanation of the general meaning of their result, or through explanation of the implications of their reclassified result for family members (Table 2). Participants with a reclassification downgrade were more likely to recall their reclassification correctly (70\%) compared to those with an upgrade (43\%). We do caution that as this is a qualitative study, the proportions described reflect the interviewed sample and not the overall population.

\section{Overview of emerging themes}

Four broad themes were identified following a variant reclassification (Fig. 1). These included patients' reaction towards the reclassified variant, the impact of the reclassified variant, patients' overall perception of receiving a reclassification, and improvement of the reclassification process.

\section{Reactions towards the reclassified variant}

Among participants that received a reclassification upgrade, relief was expressed. The relief arose from the utility of the result, as it would now allow for genetic testing to be
Table 1 Characteristics of the participants

\begin{tabular}{|c|c|}
\hline Characteristics & $n(\%)$ \\
\hline \multicolumn{2}{|l|}{ Sex } \\
\hline Female & $11(73.3)$ \\
\hline Male & $4(26.7)$ \\
\hline \multicolumn{2}{|l|}{ Ethnicity } \\
\hline Caucasian & $14(93.3)$ \\
\hline Other & $1(6.7)$ \\
\hline \multicolumn{2}{|l|}{ Age category, years } \\
\hline $20-29$ & $2(13.3)$ \\
\hline $30-39$ & $4(26.7)$ \\
\hline $40-49$ & $3(20)$ \\
\hline $50-59$ & $4(26.7)$ \\
\hline $60-69$ & $1(6.7)$ \\
\hline $70-79$ & $1(6.7)$ \\
\hline \multicolumn{2}{|l|}{ Educational level } \\
\hline Primary School & $1(6.7)$ \\
\hline High School & $5(33.3)$ \\
\hline College/University & $5(33.3)$ \\
\hline Postgraduate & $4(26.7)$ \\
\hline \multicolumn{2}{|l|}{ Diagnosed heart condition } \\
\hline Hypertrophic cardiomyopathy (HCM) & $6(40)$ \\
\hline Dilated cardiomyopathy (DCM) & $1(6.7)$ \\
\hline Left ventricular non-compaction (LVNC) & $1(6.7)$ \\
\hline $\begin{array}{l}\text { Arrhythmogenic right ventricular cardiomyopathy } \\
\text { (ARVC) }\end{array}$ & $1(6.7)$ \\
\hline Long QT syndrome (LQTS) & $4(26.7)$ \\
\hline Sudden cardiac arrest (SCA) & $2(13.3)$ \\
\hline \multicolumn{2}{|c|}{ Family history of the condition or sudden cardiac death } \\
\hline Yes & $9(60)$ \\
\hline No & $6(40)$ \\
\hline \multicolumn{2}{|l|}{ Type of reclassification } \\
\hline Upgrade $^{\mathrm{a}}$ & $6(40)$ \\
\hline Downgrade & $9(60)$ \\
\hline
\end{tabular}
aTwo participant's variants were reclassified twice, with their final
variant being upgraded to likely pathogenic

offered to the family members, or be used for reproductive planning.

"I think it's just that you know for sure... I suppose it's because the one daughter that I have is borderline and we've never been sure, it means that potentially she may be able to come off her medications." (LQTS, LP to VUS to LP)

"We decided that you know, they were confident in that... this is what's causing it. That we could go ahead with the IVF [in vitro fertilization] and doing pre-genetic testing. So, it was a relief (laughs) to know that we had a way around it." (LQTS, VUS to LP) 
Table 2 Participant recall of the reclassified genetic result

\begin{tabular}{|c|c|c|}
\hline Type of reclassification & Recall $n(\%)^{\mathrm{a}}$ & Examples \\
\hline \multirow[t]{4}{*}{ Upgrade } & Correct & \\
\hline & $3(43)$ & $\begin{array}{l}\text { "My understanding is that, the team or somebody, has confidently identified...the gene in our } \\
\text { family that is the mutation that causes the problem." (VUS to LP) }\end{array}$ \\
\hline & Incorrect/unsure & \\
\hline & $4(57)$ & $\begin{array}{l}\text { "I don't really know what it means to us because I mean I know that we, we have a problem." (VUS } \\
\text { to LP) }\end{array}$ \\
\hline \multirow[t]{4}{*}{ Downgrade } & Correct & \\
\hline & $7(70)$ & $\begin{array}{l}\text { "...the finding that my two daughters um are not um affected with the condition, might not be one } \\
\text { hundred percent accurate. That there was some ambiguity because further genetic research had um } \\
\text { yielded indications of variance..." (LP to VUS) }\end{array}$ \\
\hline & Incorrect/unsure & \\
\hline & $3(30)$ & $\begin{array}{l}\text { "...they got a new study on it that it's not um, that it's not genetic, which is a good thing." (LP to } \\
\text { VUS) }\end{array}$ \\
\hline
\end{tabular}

$L P$ likely pathogenic, $V U S$ variant of uncertain significance

${ }^{a}$ Two participants had their variant reclassified twice, and recall of both reclassifications was counted

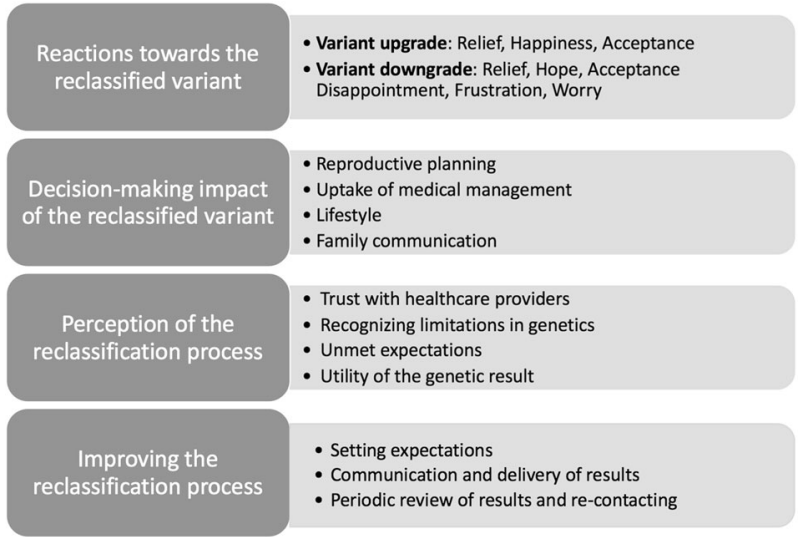

Fig. 1 A summary of emerging themes when cardiac patients experience and receive a reclassified genetic variant

Several participants reacted minimally or felt neutral towards a reclassification in their variant, with the implications of their clinical diagnosis being in the forefront. This was expressed in both reclassification groups.

"Well it doesn't really mean anything to us, we've got the heart condition, we live with it, you know?" (LVNC, VUS to LP)

"I think it's probably just left me with the sense that it's an unknown, but it hasn't changed the experience that I've been through..." (HCM, LP to VUS)

For those participants that received a reclassification downgrade to a VUS, many participants also expressed relief. This appeared to be based on a misunderstanding that a VUS meant their heart condition was less likely to be genetic, and that their family members were not at risk.
"Oh just... that it's not genetic. And ... that my kids don't have it (laughs). That's the biggest relief."

\section{(LQTS, LP to VUS)}

In contrast, some participants that received a downgrade to a VUS reacted negatively, expressing disappointment and frustration, that there was no longer an explanation for the cause of their heart condition. In others, a VUS was perceived as worrisome, as it was unclear what the variant meant for the individual and his or her family.

"Sometimes it's more frustrating... yeah, it's hurtful you know? Like they've never said why I have got this stuff." (HCM, LP to VUS)

"I suppose once you've got children, I suppose that it's the unknown, maybe. The fact that they're giving you information and they're saying that, you know... it doesn't seem like it lines up? So it doesn't seem like that's what's actually causing your problem. But I suppose [there is] the unknown that... maybe it could affect your kids." (SCA, P to VUS)

\section{Impact of the reclassified variant on decision-making}

Not all participants reported an impact from receiving a reclassified variant, though for some it affected their decision making regarding reproductive planning. Participants that received a downgrade of their variant to a VUS perceived less risk in passing the condition on to their children.

"I felt better having them [children] because, I guess when I thought that they'd find a match... I probably 
wouldn't then want to have children? But... because the match wasn't found [the variant was not identified in her affected parent], then I thought, well you know it could be perhaps that you know what, it wasn't a genetic mutation I guess". (DCM, LP to VUS)

Some participants incorrectly perceived that their reclassified variants supported their decision to not pursue, or to cease medical therapy. This was only expressed by those that received a downgrade of their variant to a VUS.

"All of them [doctors]... they said to put [in] the defibrillator. But you know, as I mentioned, I knew there was something, a gut feeling of why do I need that? I've never felt this great... Now that I know it's uncertain significance, I can be on a positive side." (HCM, LP to VUS)

"I stopped taking them [beta-blockers] a while ago, which is good, because I didn't even need to really take them right?" (LQTS, LP to VUS)

One participant noted an impact to their lifestyle. When the variant was downgraded to a VUS, this appeared to lead the participant to perceive less risk and feel safer in engaging in physical activities.

"I would be yeah, more likely to engage in sports activities... it was just... the mental barriers to doing something, were removed." (LQTS, LP to VUS)

Interestingly, a reclassification appeared to have an impact on family communication. One participant felt that it was unnecessary to disclose their downgraded variant to distant family members, and in not disclosing this would avoid unnecessary anxiety.

"I think that... until we have something more concrete, [I] just decided to just leave them as they are. And you know, they are really up to date on what the symptoms could be if any of their kids have anything. But as far as they're concerned... we think we know why I have it, which is not exactly true." (ARVC, LP to VUS)

\section{Perception of the reclassification process}

None of the participants expressed major dissatisfaction with the process that led to their variant being reclassified over time.
"I look at science as not necessarily being the most... I say science, but I think it's medicine, right, it's genetics. Anyway... research takes time and things that are unknown, need to be figured out. And it doesn't just happen overnight. So I just, you know, patience in this sort of thing is required." (LQTS, LP to VUS)

Moreover, participants' preexisting relationship with their health providers was an important influence on their views of the reclassification process. In fact, several participants commented that the delivery of their reclassified result strengthened their trust with their health providers.

"uh yeah it actually increased my confidence in the medical system" (HCM, LP to VUS)

"The fact that they delivered this extra news, I guess, is just for me, proof that they've been continuing to keep working to try and find answers. And at all times they've communicated and kept us in the loop as much as they could." (LQTS, LP to VUS to LP)

However, some participants expressed disappointment throughout the reclassification process due to their expectations being unmet. This ranged from inappropriate expectations following pre-test counseling, to expectations about the follow-up process after a reclassification.

"I think all my doctors have kind of said "you know... we know it's genetic, and you know we recommend that you do this testing" and, so I just... had an expectation that something would be found" (DCM, LP to VUS)

"the only issue I had was more so that there wasn't a clear-cut kind of concession plan put in place once that downgrade happened... we're basically just going to park this and so you know, just reconvene in five years." (HCM, P to B)

In all cases, participants did not express regret for undergoing genetic testing, and still valued the process despite having undergone a reclassification of their variant. This arose from the perceived importance of the reclassified genetic result for immediate family members. Others were altruistic, stating it was relevant for future generations even if the result lacked personal utility.

"If they can't find anything, it's a better result for my family, and at the end of the day, no matter who it was delivering that news, that's good news." (SCA, P to VUS) 
"I never really understand why people are kind of on the fence about it... even if it doesn't affect things directly for you, it could affect things for your relatives in the future, or just for other people in the future." (ARVC, LP to VUS)

\section{Improving the reclassification process}

Many participants expressed satisfaction despite having undergone the process of having their variant reclassified. However, setting expectations in terms of communication during the reclassification process was important to some participants.

"I do think communication throughout the process needs to maybe be a little bit more consistent. So, I mean look, I'm not saying that I want a phone call every week just to say hello. What I'm asking is, is that if there are any changes, or if there's changes in the process, just to remain updated."

\section{(HCM, P to B)}

Participants generally felt that the reclassified result was explained clearly, and had no strong preference with the mode of delivery (telephone or in person). A few participants preferred their reclassified result to be disclosed by their multidisciplinary cardiology team, as they had a longstanding relationship with them.

"I think it was actually a pretty good experience. It was delivered from a team that I trust and I really liked and really value... so the best thing you could have was that it was being delivered by that team, not really by someone who doesn't really know you or understand your personal situation" (SCA, P to VUS)

"I like the idea of having everybody [cardiology and genetics] together in one room... because it kind of shows a bit of a united front in terms of my health care, and my health care providers. And I like the idea of knowing that they're talking amongst themselves as well." (ARVC, LP to VUS)

Regarding the responsibility for review of genetic results and re-contacting, the majority of the participants felt this should be a health provider-driven process. Many expressed that it was not reasonable for patients to periodically call their health provider for an update on the status of their variant. Others assumed their variants were periodically reviewed, and if no communication had been made by the health provider, then no reclassification had occurred. However, some participants acknowledged that it would not be practical for health providers to re-contact all of their patients, and felt that it was a shared responsibility, providing the health provider sets appropriate expectations.

"I think it is on the health care provider to let you know. Because if I were to call once a year, they wouldn't like that much. Right, and me times a thousand, times ten thousand... that doesn't even seem like it's... plausible." (SCA, VUS to VUS/LP)

"No, I wouldn't [contact the health provider]. I feel that if they found something exciting they would get in touch with me." (HCM, VUS to LP)

"...If she had said, "you know, I would welcome your calling every year, cause it's hard for me to do that. And if you checked in and remind me...". Cause I mean I have no idea how many... patients she has. I assume quite a bunch... So yeah, if we were invited to do that, I would be happy to do that." (HCM, LP to VUS to LP).

\section{Discussion}

We found that patients frequently demonstrated confusion and misunderstanding about the meaning and implications of their reclassified cardiac genetic results. A variant upgrade was often received positively, with patients expressing relief and happiness. A downgrade was in many cases considered "good news", with an incorrect belief that their condition was no longer familial, despite being seen in centers with specialized multidisciplinary expertise. In the current climate of increasingly comprehensive genetic testing, the inherent uncertainty of a genetic result must be considered. A reclassification can be due to changing knowledge, an error, or misuse of the variant interpretation criteria. As such, the uncertainty of a genetic result and the possibility of a variant being reclassified over time should be raised with patients during pre-test counseling and consent. As well, it will be important to assess patient understanding and establish expectations towards genetic testing to minimize frustration when being recontacted. More effective methods to support our discussion with patients about uncertainty and the potential for reclassification are crucial to ensure a deeper understanding of the outcomes of genetic testing and its impact on the family.

A variant downgrade can lead to potential harms, with first-degree relatives having been inappropriately followed or released from clinical surveillance. The fear of over- 
zealous classification of variants later shown to be benign is a key driver in the recent tightening of the widely used ACMG/AMP criteria. Case examples from the literature provide a stark illustration of the potential for harm when a variant is incorrectly classified. One example includes family members who have been misdiagnosed with an inherited arrhythmia syndrome on the basis of testing gene positive, with some inappropriately receiving an implantable cardioverter defibrillator [14]. A variant downgrade could cause harm via patient misunderstanding. As mentioned above, our participants perceived a variant downgrade to be good news, suggesting that they understood the disease to no longer be familial. Uncertainty can lead to greater likelihood of a misunderstanding of a result, with one study showing VUS genetic results were frequently misinterpreted [10]. Importantly, a misunderstanding is likely to impair subsequent communication to the at-risk relatives [15]. Amongst our participants, one suggested that the reclassification downgrade had impacted family communication, whereby they deliberately chose not to disclose the revised result to relatives given the perceived lack of utility. Future research should aim to address the influence of reclassification on family communication.

A study investigating experiences with receiving uninformative variant results in patients with LQTS found some participants expressed disappointment and frustration [16]. This is consistent with the view that genetic testing is pursued mainly to clarify risks for family members but also to provide an explanation for the cause of their condition [17]. Despite this, we found some participants still perceived value in genetic testing even when there was no personal utility in their reclassified result, as they emphasized its importance for future generations or to advance research. Confidence in the medical system was a strong theme we observed, with participants acknowledging the current limitations in our genetic knowledge that led to the reclassification. We show that for the most part, participants did not regret their decision to undergo genetic testing even in spite of the reclassification, though confirmation bias may be an important driver of this finding.

There is a lack of consensus among the medical community regarding the duty to re-contact. A study that surveyed clinical genetics providers in the UK found that majority of genetic centers only re-contacted patients when there was an event that prompted clinicians to review their file [18]. Another study investigated the views of various health providers on re-contacting and found that some expected patients to share the responsibility by initiating contact first [19]. We show that many patients make the assumption that their health provider will periodically review their results and provide updates, whereas health providers may expect patients to initiate contact first given their limited time and resources. Such a misunderstanding could lead to delays in new information about a variant being formally considered, with potential implications for the family. Our results illustrate the need for genetic health providers to establish clearer expectations with patients on the reclassification process and the logistics of initiating recontacting during post-test counseling. While some patients agreed they would be willing to take a more proactive role in initiating periodic variant reassessment, this may not be practical in reality. Given the potential complexity and nuances of variant interpretation, these results support the recommendation that patients with inherited cardiac conditions should be followed long-term in a multidisciplinary clinic with genetics expertise embedded [1, 20, 21]. Such a clinic model has the expertise in accurate variant interpretation and provides a mechanism for a periodic reassessment of variant pathogenicity when families attend their routine follow-up. Indeed, a recent study showed genetic counselors are integral to variant work up in a cardiology setting, thus highlighting the importance for their inclusion in a multidisciplinary team [22]. Considering many patients are not managed in specialized multidisciplinary clinics, other avenues to improve the process of periodic reclassification should be explored. For example, some have suggested clinical genetic testing laboratories as the initiator of the reclassification process, given their access to information that may change the interpretation of the variant [23]. Alternatively, the creation of automated and centralized variant sharing platforms should also be considered.

Based on our results, we highlight key discussion points to be raised with patients during pre- and post-test genetic counseling, that may assist should a variant reclassification arise in future (Fig. 2). Specifically, genetic health providers should raise the possibility of a variant reclassification by

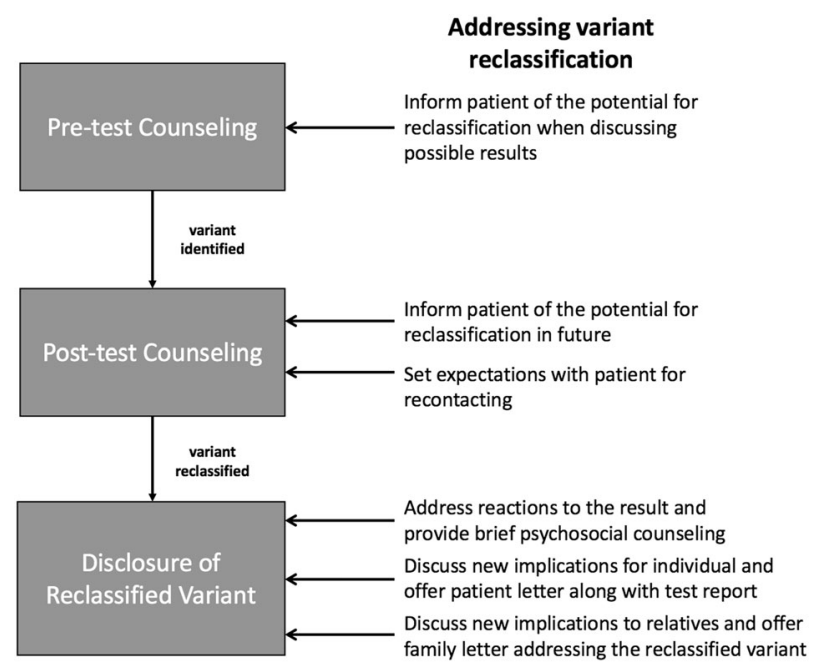

Fig. 2 Recommendations for incorporating the variant reclassification discussion throughout the different stages of genetic counseling 
emphasizing the evolving nature of the evidence used to interpret genetic variants. We recommend establishing clear expectations with the patient on the process of formal review of the variant, and this will be specific to the resources of different centers with some classifying themselves while others may rely on notification from the clinical laboratory. In the event of a reclassification, information and support to adequately convey this new information to relatives are needed, given the potential impact on their surveillance and medical recommendations. Our findings reiterate the critical need for diagnostic laboratories to remain vigilant in their processes for identifying and reporting genetic variants, as well as the importance of continued research efforts to elucidate the genetic architecture of cardiac genetic diseases.

\section{Conclusion}

We describe the experience of receiving a genetic variant reclassification in a cardiac genetic setting. While patients did not report any overt long-term psychosocial impact as a result of a receiving a variant reclassification, we did show a failure to correctly understand the meaning of their reclassified variant, with potential for impact on family communication and cascade screening of at-risk relatives. We also illustrate the expectation of patients that their health providers will routinely reassess variant classifications and notify them of changes. Our findings raise important counseling points that should be addressed with patients during genetic counseling. This includes a discussion of patient understanding and expectations regarding genetic testing results and the possibility of reclassification during pre-test counseling, as well as the responsibility of periodic variant review and recontacting during post-test counseling. The role of the specialized multidisciplinary clinic is key in ensuring timely and accurate variant interpretation, and access to experienced cardiac genetic counseling.

Acknowledgements $\mathrm{CB}$ is the recipient of an Australia Postgraduate Award (APA). CS is the recipient of a National Health and Medical Research Council (NHMRC) Practitioner Fellowship (\#1059156). ADK receives support from the Heart and Stroke Foundation of Canada (Ottawa, ON), the Sauder Family and Heart and Stroke Foundation Chair in Cardiology (Vancouver, BC), the Paul Brunes Chair in Heart Rhythm Disorders (Vancouver, BC), and the Paul Albrechtson Foundation (Winnipeg, MB). JI is a recipient of a Heart Foundation of Australia Future Leader Fellowship (\#100833).

\section{Compliance with ethical standards}

Conflict of interest The authors declare that they have no conflict of interest.
Publisher's note: Springer Nature remains neutral with regard to jurisdictional claims in published maps and institutional affiliations.

\section{References}

1. Ackerman MJ, Priori SG, Willems S, Berul C, Brugada R, Calkins $\mathrm{H}$, et al. HRS/EHRA expert consensus statement on the state of genetic testing for the channelopathies and cardiomyopathies this document was developed as a partnership between the Heart Rhythm Society (HRS) and the European Heart Rhythm Association (EHRA). Heart Rhythm. 2011;8:1308-39.

2. Burns C, Bagnall RD, Lam L, Semsarian C, Ingles J. Multiple gene variants in hypertrophic cardiomyopathy in the era of nextgeneration sequencing. Circ Cardiovasc Genet. 2017;10: e001666.

3. Alfares AA, Kelly MA, McDermott G, Funke BH, Lebo MS, Baxter SB, et al. Results of clinical genetic testing of 2,912 probands with hypertrophic cardiomyopathy: expanded panels offer limited additional sensitivity. Genet Med. 2015;17:880-8.

4. Ingles J, Semsarian C. Conveying a probabilistic genetic test result to families with an inherited heart disease. Heart Rhythm. 2014;11:1073-8.

5. Richards S, Aziz N, Bale S, Bick D, Das S, Gastier-Foster J, et al. Standards and guidelines for the interpretation of sequence variants: a joint consensus recommendation of the American College of Medical Genetics and Genomics and the Association for Molecular Pathology. Genet Med. 2015;17:405-24.

6. Lek M, Karczewski KJ, Minikel EV, Samocha KE, Banks E, Fennell T, et al. Analysis of protein-coding genetic variation in 60,706 humans. Nature. 2016;536:285-91.

7. Schymanski R, Sturm A, Hashimoto S, Morales A, Pyatt R. Impact of variant reclassification in the clinical setting of cardiovascular genetics. J Genet Couns. 2017;26:1411-511. (abstract)

8. Das KJ, Ingles J, Bagnall RD, Semsarian C. Determining pathogenicity of genetic variants in hypertrophic cardiomyopathy: importance of periodic reassessment. Genet Med. 2014;16:28693.

9. Murray ML, Cerrato F, Bennett RL, Jarvik GP. Follow-up of carriers of BRCA1 and BRCA2 variants of unknown significance: variant reclassification and surgical decisions. Genet Med. 2011;13:998-1005.

10. Burns C, Yeates L, Spinks C, Semsarian C, Ingles J. Attitudes, knowledge and consequences of uncertain genetic findings in hypertrophic cardiomyopathy. Eur J Hum Genet. 2017; 25:809-15.

11. Solomon I, Harrington E, Hooker G, Erby L, Axilbund J, Hampel $\mathrm{H}$, et al. Lynch syndrome limbo: patient understanding of variants of uncertain significance. J Genet Couns. 2017;26:866-77.

12. Makhnoon S, Garrett LT, Burke W, Bowen DJ, Shirts BH. Experiences of patients seeking to participate in variant of uncertain significance reclassification research. J Community Genet. 2018. https://doi.org/10.1007/s12687-018-0375-3.

13. Creswell JW. Qualitative inquiry and research design: choosing among five approaches. 3rd edn. Thousand Oaks, CA, USA: Sage Publications; 2003.

14. Ackerman JP, Bartos DC, Kapplinger JD, Tester DJ, Delisle BP, Ackerman MJ. The promise and peril of precision medicine: phenotyping still matters most. Mayo Clin Proc. 2016;91: $1606-16$.

15. Burns C, James C, Ingles J. Communication of genetic information to families with inherited rhythm disorders. Heart Rhythm. 2018;15:780-6. 
16. Predham S, Hathaway J, Hulait G, Arbour L, Lehman A. Patient recall, interpretation, and perspective of an inconclusive long QT syndrome genetic test result. J Genet Couns. 2017;26:150-8.

17. Khouzam A, Kwan A, Baxter S, Bernstein JA. Factors associated with uptake of genetics services for hypertrophic cardiomyopathy. J Genet Couns. 2015;24:797-809.

18. Carrieri D, Lucassen AM, Clarke AJ, Dheensa S, Doheny S, Turnpenny PD, et al. Recontact in clinical practice: a survey of clinical genetics services in the United Kingdom. Genet Med. 2016;18:876-81.

19. Carrieri D, Dheensa S, Doheny S, Clarke AJ, Turnpenny PD, Lucassen AM, et al. Recontacting in clinical practice: an investigation of the views of healthcare professionals and clinical scientists in the United Kingdom. Eur J Hum Genet. 2017;25: $275-9$.
20. Hershberger RE, Givertz MM, Ho CY, Judge DP, Kantor PF, McBride KL et al. Genetic evaluation of cardiomyopathy-a Heart Failure Society of America practice guideline. J Card Fail. 2018;24:281-302.

21. Ingles J, Lind JM, Phongsavan P, Semsarian C. Psychosocial impact of specialized cardiac genetic clinics for hypertrophic cardiomyopathy. Genet Med. 2008;10:117-20.

22. Reuter C, Grove ME, Orland K, Spoonamore K, Caleshu C. Clinical cardiovascular genetic counselors take a leading role in team-based variant classification. J Genet Couns. 2017;27:751-60.

23. Carrieri D, Howard HC, Benjamin C, Clark AJ, Dheensa S, Doheny $\mathrm{S}$, et al. Recontacting patients in clinical genetics services: recommendations of the European Society of Human Genetics. Eur J Hum Genet. 2018. https://doi.org/10.1038/s41431018-0285-1. 